\title{
REVIEW
}

\section{Modifiable influences on female HPV vaccine uptake at the clinic encounter level: A literature review}

Stephanie L. Small, PhD, WHNP-BC, ANP-BC (Nurse Practitioner) ${ }^{1}$, Carolyn M. Sampselle, PhD, ANP (Professor) ${ }^{1}$, Kristy K. Martyn, PhD, FNP-BC, CPNP-PC (Associate Professor) ${ }^{1}$, \& Amanda F. Dempsey, MD, PhD, MPH

(Associate Professor) $)^{2}$

${ }^{1}$ University of Michigan School of Nursing, Ann Arbor, Michigan

${ }^{2}$ University of Colorado, Denver, Colorado

\author{
Keywords \\ Human papilloma virus (HPV); vaccination; \\ immunization; prevention.

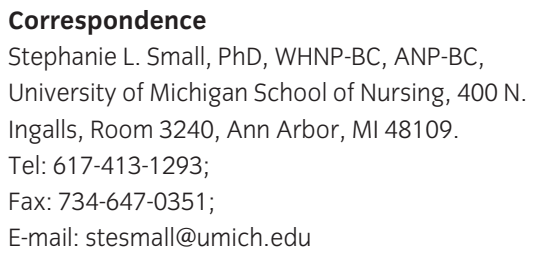

\begin{abstract}
Purpose: A review of the literature to identify modifiable influences on female human papillomavirus (HPV) vaccine uptake relevant to clinical practice in order to support nurse practitioners (NPs) in the prevention of cervical cancer.

Data sources: PubMed, CINAHL, reference lists of publications that surfaced in the electronic search.

Conclusions: Six influences are modifiable and potentially amenable to being addressed at the clinic encounter level: (a) cost and insurance coverage, (b) provider recommendation, (c) vaccination opportunity, (d) HPV and HPV vaccine knowledge, (e) vaccine safety concerns, and (f) HPV risk.

Implications for practice: NPs have an important role in improving HPV vaccine uptake and research suggests several areas they can address to increase vaccination during clinic visits.
\end{abstract}

\section{Introduction}

Infection with human papillomavirus (HPV) is the established cause of most cervical cancers (Bosch, Lorincz, Munoz, Meijer, \& Shah, 2002) and costs the U.S. health system 5 billion dollars a year in direct medical costs (Insinga, Dasbach, \& Elbasha, 2005). In the United States, $25 \%$ of 14 - to 19 -year-old females, $45 \%$ of 20 - to 24 year-old females, and $27 \%$ of 25 - to 29 -year-old females are infected with HPV (Dunne et al., 2007). The Advisory Committee on Immunization Practices (ACIP) recommends universal HPV vaccination for all females (since 2006) and males (since 2011) starting at age 11-12. Acceptability of the vaccine is high $(55 \%-100 \%)$ among adolescents, young adults, and parents of adolescents (Brewer \& Fazekas, 2007). However, only $49 \%$ of adolescent females have initiated the series and only $32 \%$ have received all three doses required for full immunization (Centers for Disease Control and Prevention, 2011). Even fewer young adult females have started the series, with national estimates at $17 \%$ (Centers for Disease Control and Prevention, 2010).

Nurse practitioners (NPs) play a vital role in making sure their patients receive cancer protection through vac- cination, but may not have a clear understanding about what areas they can address with patients to improve vaccination. This literature review was conducted to identify modifiable influences on female HPV vaccine uptake relevant to clinical practice in order to support NPs in the prevention of cervical cancer.

\section{Methods}

An electronic search was conducted in PubMed using the search term "hpv vaccine." The search was limited to humans and female participants, as routine recommendation for HPV vaccination was limited to females until October 2011, and the search was limited to publications in English, as the journals reporting results in the United States are published in English. The search was also limited to articles published between January 1, 2009 and June 1, 2011, the time period when researchers began publishing HPV vaccine uptake studies. CINAHL was searched separately using the same search term and time limitations to identify any additional studies not available in PubMed. Hand searches were also performed of reference lists of publications that surfaced in the electronic 
search and met the inclusion criteria, and the first author added her own publication.

Studies were included if they provided direct measurement of uptake (not simply intention to vaccinate) and used variables the investigator identified as modifiable in clinical practice (such as costs, recommendations, opportunity, knowledge, and risk). Studies were excluded if they examined variables unable to be modified directly in clinical practice, such as race or ethnicity, age, education level, income, marital status, or number of previous hospitalizations. Studies were also excluded if they were not conducted in the United States, in order to increase relevance to clinical practice within the United States. Each study was analyzed to identify the main findings. Similar variables from the main findings were grouped together to extract summary factors of modifiable influences at the clinic encounter level.

\section{Results}

Of the 1074 articles identified through the electronic database search, a total of 19 articles met the inclusion criteria. Table Sl summarizes the findings from these studies. Six modifiable influences relevant to clinical practice were extracted from the studies (see Table 1). The six influences were (a) cost and insurance coverage, (b) provider recommendation, (c) vaccination opportunity, (d) HPV and HPV vaccine knowledge, (e) vaccine safety concerns, and (f) HPV risk (including documented HPV risk factors or perceived HPV risk).

\section{Cost and insurance coverage}

Cost and insurance coverage is potentially modifiable at the clinic encounter in that organizations may have access to resources for patients who cannot afford to pay. Cost and insurance coverage in general differs greatly between females younger than 19 years of age, who are more often insured and may be eligible for free vaccines through the Vaccines for Children program, and females 19 and older who are less commonly insured and have limited access to vaccine-specific funding. Of the seven studies that addressed cost and insurance coverage as influences on HPV vaccination (Caskey, Lindau, \& Alexander, 2009; Conroy et al., 2009; Dempsey, Cohn, Dalton, \& Ruffin, 2009; Jain et al., 2009; Moore, Crosby, Young, \& Charnigo, 2010; Schluterman, Terplan, Lydecker, \& Tracy, 2011; Zimet, Weiss, Rosenthal, Good, $\delta$ Vichnin, 2010), most found cost and insurance coverage to be an influence in the young adult age group.

Across the eligible age range, in a longitudinal survey of 189 females 13- to 26-year-old, Conroy et al.
Table 1 HPV vaccine uptake studies by influence

\begin{tabular}{|c|c|}
\hline Influence & Study \\
\hline \multirow[t]{7}{*}{ Cost and insurance coverage } & Caskey et al. (2009) \\
\hline & Conroy et al. (2009) \\
\hline & Dempsey et al. (2010) \\
\hline & Jain et al. (2009) \\
\hline & Moore et al. (2010) \\
\hline & Schluterman et al. (2011) \\
\hline & Zimet et al. (2010) \\
\hline \multirow[t]{7}{*}{ Provider recommendation } & Caskey et al. (2009) \\
\hline & Conroy et al. (2009) \\
\hline & Dempsey et al. (2009) \\
\hline & Gerend et al. (2009) \\
\hline & Gottlieb et al. (2009) \\
\hline & Guerry et al. (2011) \\
\hline & Rosenthal et al. (2011) \\
\hline \multirow[t]{6}{*}{ Vaccination opportunity } & Caskey et al. (2009) \\
\hline & Chao et al. (2010) \\
\hline & Cook et al. (2010) \\
\hline & Dempsey et al. (2010) \\
\hline & Reiter et al. (2010) \\
\hline & Small and Patel (2012) \\
\hline \multirow[t]{8}{*}{ HPV and HPV vaccine knowledge } & Brewer et al. (2011) \\
\hline & Caskey et al. (2009) \\
\hline & Gerend et al. (2009) \\
\hline & Gottlieb et al. (2009) \\
\hline & Guerry et al. (2011) \\
\hline & Licht et al. (2010) \\
\hline & Mathur et al. (2010) \\
\hline & Zimet et al. (2010) \\
\hline \multirow[t]{3}{*}{ Vaccine safety concerns } & Dempsey et al. (2009) \\
\hline & Gerend et al. (2009) \\
\hline & Zimet et al. (2010) \\
\hline \multirow[t]{8}{*}{ HPV risk } & Caskey et al. (2009) \\
\hline & Chao et al. (2010) \\
\hline & Cook et al. (2010) \\
\hline & Dempsey et al. (2009) \\
\hline & Gottlieb et al. (2009) \\
\hline & Licht et al. (2010) \\
\hline & Moore et al. (2010) \\
\hline & Zimet et al. (2010) \\
\hline
\end{tabular}

Note. HPV, human papillomavirus.

(2009) identified insurance coverage for the vaccine as the strongest predictor of HPV vaccine initiation (odds ratio [OR] 5.31, 95\% confidence interval [CI] 1.6117.49). Demonstrating the difference between the two age groups, $27 \%$ of 18 - to 26-year-old females cited cost as a reason for foregoing vaccination, while only $10 \%$ of younger females ages 13-17 years cited cost as a barrier ( $n=1011$; Caskey et al., 2009).

Among studies specific to young adults, who are therefore no longer eligible for the Vaccines for Children program, in an analysis of the 2007 National Immunization Survey-Adult data, Jain et al. (2009) found having health 
insurance as one of the only variables associated with initiation $(p<.05, n=168)$. In another study of 19- to 26-year-old insured females, $24.4 \%$ cited uncertainty about insurance coverage as a reason for not vaccinating $(n=185$; Zimet et al., 2010).

Even the type of insurance seems to have an effect on vaccination. In a study of outpatient gynecologic clinics, $47 \%$ of the 9- to 26-year-old females who initiated the HPV vaccine series had public insurance compared to $28 \%$ with private insurance $(p<.01, n=$ 8069; Schluterman et al., 2011). This finding was replicated in young adults specifically, where having public insurance increased the likelihood of vaccination initiation when compared to private insurance (OR 0.52, 95\% CI $0.45-0.59$ ) or no insurance (OR 0.47 , 95\% CI $0.26-$ $0.85, n=10,082$; Dempsey et al., 2010). However, the reverse was true when assessing vaccine series completion, with privately insured individuals more likely than publicly insured individuals to complete all three doses.

One study evaluated the impact of eliminating cost as a barrier by providing a free vaccine voucher at a university health center. This was the only study that provided an intervention to evaluate the effect of cost as an influence. As a result, $50 \%$ of the participants $(n=209)$ utilized the voucher to initiate vaccination (Moore et al., 2010).

\section{Provider recommendation}

Perhaps the most clinically relevant factor that has repeatedly been shown to influence HPV vaccine uptake is provider recommendation. Seven studies confirmed that discussing the vaccine and receiving a recommendation from a healthcare provider often results in receipt of the vaccine (Caskey et al., 2009; Conroy et al., 2009; Dempsey, Abraham, Dalton, \& Ruffin, 2009; Gerend, Weibley, \& Bland, 2009; Gottlieb et al., 2009; Guerry et al., 2011; Rosenthal et al., 2011). One survey of 19- to 26-year-old females, in which all participants were insured, found even the strength of the physician recommendation mattered, with patients who perceived a stronger recommendation from their physician having a four times greater likelihood of vaccinating than those who perceived a weaker recommendation from their physician ( $n=530$; Rosenthal et al., 2011).

While less modifiable but still important to understand, the specialty of the provider may be influential. One study of Kaiser Permanente's immunization data found that having a pediatrician as a primary care provider was correlated with vaccine initiation when compared to family medicine providers (RR $0.81,95 \%$ CI $0.79-0.83$ ) or internal medicine providers (RR 0.93, 95\% CI 0.87-1.00, $n=285,265$; Chao, Velicer, Slezak, \& Jacobsen, 2010).
A larger study of clinic visit data confirmed the influence of provider specialty where pediatric providers were positively correlated with HPV vaccination when compared to family medicine (OR $0.92,95 \%$ CI 0.81-1.04) or gynecology providers (OR $0.24,95 \%$ CI $0.18-0.33, n=10,082$; Dempsey et al., 2010). These findings are consistent with uptake among other vaccines, not just the HPV vaccine, showing pediatricians consistently outperforming other provider specialties in vaccine administration.

Provider characteristics other than specialty may also influence vaccine uptake. Having a male primary care provider was inversely associated with vaccine initiation (RR 0.92, 95\% CI 0.91-0.93, $n=285,265$ ) when compared to female primary care providers (Chao et al., 2010). Also, one of the first studies to explore influences on mothers' decisions to vaccinate daughters found that mothers who declined vaccination at a healthcare visit had more often seen someone other than their usual healthcare provider $(n=52)$. This supports the belief that the strength of the relationship between provider and patient also matters, and not just the provider specialty or gender (Dempsey et al., 2009).

\section{Vaccination opportunity}

Four studies found that females with a recent healthcare visit were more likely to have received the HPV vaccine than those without a recent healthcare visit (Caskey et al., 2009; Chao et al., 2010; Dempsey et al., 2010; Reiter et al., 2010), as the healthcare visit serves as an opportunity to access the vaccine. A total of $13 \%$ of parents interviewed in North Carolina cited the lack of a healthcare visit as the reason they had not initiated vaccination for their daughters ( $n=889$; Gottlieb et al., 2009). However, the frequency of healthcare visits did not seem to be associated with vaccination ( $n=177$; Mathur, Mathur, $\delta$ Reichling, 2010). Females who attended an outpatient visit ( $n=718,660$; Cook et al., 2010) and females who attended a preventive maintenance visit (OR 5.18, 95\% CI 4.64-5.79, $n=10,082$; Dempsey et al., 2010) were more likely to initiate HPV vaccination than those attending a problem-focused visit. This finding supports the understanding that vaccination opportunity specifically, rather than healthcare visits generally, influence vaccination, as preventive maintenance visits are traditionally when vaccination status is addressed.

One study showed that having the vaccine available at a clinic visit makes a difference. Four years after the vaccine became available to the public, vaccine availability at a clinic visit increased vaccination from $11.1 \%$ prior to health center availability to $17.0 \%$ after it became available at the health center ( $n=171$; Small \& Patel, 2012). 


\section{HPV and HPV vaccine knowledge}

Knowledge about HPV and HPV vaccines are often modifiable at the clinic encounter as health centers have the opportunity to educate patients and parents about the common virus and vaccine protection available. Eight studies examined HPV and HPV vaccine knowledge, as well as the source of that knowledge, as an influence on vaccination (Brewer et al., 2011; Caskey et al., 2009; Gerend et al., 2009; Gottlieb et al., 2009; Guerry et al., 2011; Licht et al., 2010; Mathur et al., 2010; Zimet et al., 2010). The results were mixed of how important these influences are on vaccine uptake, although knowledge never dissuaded vaccination. Differences may vary among parents of adolescents, whose are deciding whether or not to vaccinate their child, and young adults, who are often deciding to vaccinate themselves.

Among studies of parents of adolescents, knowledge of the HPV vaccine (but not HPV) was correlated with uptake in a small $(n=82)$ study of parents $(t-3.214$, $p<.01$; Gerend et al., 2009). In an early survey of parents, $14 \%$ had not heard of the vaccine $(13 \%$ had not seen a healthcare provider) and the most commonly cited $(22 \%)$ reason for not initiating was the need for more information ( $n=889$; Gottlieb et al., 2009). This study was conducted only a year after the HPV vaccine was approved and only $10 \%$ of those surveyed had initiated vaccination. Two later studies supported the finding that needing more information about the HPV vaccine decreased the likelihood of vaccine initiation (adjusted odds ratio [aOR] 0.08, 95\% CI 0.04-0.2, $n=509$; Guerry et al., 2011) and not needing more information increased the likelihood of initiation (adjusted risk ratio [aRR] 0.41, 95\% CI 0.22-0.76, $n=650$; Brewer et al., 2011).

Among young adults, in a study of 406 university females, participants who knew that HPV caused genital warts were more likely to be vaccinated (aOR 1.85, 95\% CI 1.20-2.93, $n=406$; Licht et al., 2010). Two years after vaccine availability, $31.7 \%(n=185)$ of females reported lack of information about the vaccine as a reason for not initiating vaccination (Zimet et al., 2010).

The source of vaccine information may also influence vaccine uptake across the eligible age range. A large, nationally representative survey $(n=1011)$ found that vaccinated females were more likely to identify healthcare providers as their source of HPV vaccine information $(69 \%)$ than unvaccinated females $(28 \%)$, and $77 \%$ of all participants identified their healthcare provider as the source of HPV information they trust most (Caskey et al., 2009). This study did not differentiate types of healthcare providers. One survey of 177 high school females in California asked about the source of HPV vaccine knowledge and found that learning about the HPV vaccine from a physician or nurse was associated with vaccination (Mathur et al., 2010).

\section{Vaccine safety concerns}

Three studies indicated that vaccine safety concerns influence vaccine uptake (Dempsey et al., 2009; Gerend et al., 2009; Zimet et al., 2010). A study interviewing mothers of 11 - to 17-year-old females who attended a recent healthcare visit found 8 of the 19 mothers of unvaccinated daughters cited safety concerns because of its recent availability as a reason for declining vaccination (Dempsey et al., 2009). Even in mothers who chose to vaccinate, 7 of 33 expressed concerns about safety, but felt the benefits outweighed the risks (Dempsey et al., 2009). In 19- to 26-year-old females less than 2 years after vaccine approval, participants reported the newness of the vaccine $(35.4 \%)$ and side effect concerns $(24.4 \%)$ as reasons for not initiating vaccination $(n=185$; Zimet et al., 2010). In a survey also approximately 2 years after HPV vaccine availability, $24 \%$ of parents $(n=82)$ indicated the vaccine would have to be on the market for more than 5 years before they would feel comfortable providing it for their daughter (Gerend et al., 2009).

\section{HPV risk}

Eight studies examined HPV risk as an influence on HPV vaccination, either through documented risk factors or risk perception (Caskey et al., 2009; Chao et al., 2010; Cook et al., 2010; Dempsey et al., 2009; Gottlieb et al., 2009; Licht et al., 2010; Moore et al., 2010; Zimet et al. 2010). Two studies examined clinical risk factors for HPV. Chao et al. (2010) found in 18- to 26-year-old females that risk factors for HPV, including history of sexually transmitted infections, abnormal Pap smears, and oral and transdermal contraceptive use are associated with HPV vaccine uptake ( $n=285,265)$. This study examined a mostly insured population and did not investigate why the association exists. Conversely, a study of university females found that sexual activity in the past 12 months, a history of a Pap smear or abnormal Pap smear, or a history of a sexually transmitted infection was not associated with vaccination ( $n=209$; Moore et al., 2010).

Six of the studies examined HPV risk perception. Mothers $(n=52)$ who declined vaccination for their daughters cited low HPV risk as a contributing factor in their decision, and those who chose vaccination protection for their daughters recognized high risk of HPV as motivating their choice (Dempsey et al., 2009). In a larger study ( $n$ $=889$ ), $12.6 \%$ of parents with unvaccinated daughters cited the belief that their daughter was not yet sexually active as a reason for not initiating the vaccine (Gottlieb 
Table 2 Implications for practice

\begin{tabular}{|c|c|}
\hline Influence & Recommendation \\
\hline Cost and insurance coverage & $\begin{array}{l}\text { For clinicians of patients under age 19, providers should enroll in the Vaccines for Children program, } \\
\text { enabling patients who are Medicaid eligible, uninsured, underinsured, American Indian or Alaska Native } \\
\text { to receive free vaccine (www.cdc.gov/vaccines/programs/vfc/). Patients } 19 \text { and older without health } \\
\text { insurance coverage who meet certain income guidelines may qualify for free vaccines through the Merck } \\
\text { Vaccine Patient Assistance Program (www.merck.com/merckhelps). }\end{array}$ \\
\hline Provider recommendation & $\begin{array}{l}\text { All clinicians should recognize their own influence on patients' decisions to vaccinate, regardless of } \\
\text { specialty, and responsibly convey vaccine recommendations consistent with evidence-based guidelines. }\end{array}$ \\
\hline Vaccination opportunity & Clinicians should supply the HPV vaccine, recognizing that any visit may be their patients' only opportunity. \\
\hline HPV and HPV vaccine knowledge & $\begin{array}{l}\text { Clinicians should provide accurate, evidence-based information about HPV and the HPV vaccine to all } \\
\text { patients eligible for vaccination. }\end{array}$ \\
\hline Vaccine safety concerns & $\begin{array}{l}\text { Should safety concerns arise, clinicians can reassure patients that ongoing postlicensure safety surveillance } \\
\text { and } 23 \text { million doses distributed continue to confirm very high vaccine safety (Slade et al., 2009). }\end{array}$ \\
\hline HPV risk & $\begin{array}{l}\text { Clinicians should reinforce the message that the HPV vaccine is indicated as a routine immunization, not risk } \\
\text { based. }\end{array}$ \\
\hline
\end{tabular}

et al., 2009). Thirty percent of 13- to 26-year-old females also reported their lack of sexual activity as a reason for not vaccinating ( $n=1011$; Caskey et al., 2009). A study of Florida Medicaid patients $(n=718,660)$ found sexual activity to be positively associated with vaccine initiation (OR 1.19, 95\% CI 1.15-1.24; Cook et al., 2010). However, another study of university females found that risk perception (Licht et al., 2010) had no association with vaccination $(n=406)$. In a study of 19 - to 26 -year-old females $(n=185)$, the most commonly cited reason for not initiating vaccination was the belief that they were in a monogamous relationship (Zimet et al., 2010).

\section{Discussion}

Because of the relatively recent availability of HPV vaccines, research is still in the early stages of evaluating uptake. However, a review of 19 available studies exploring HPV vaccine uptake did reveal six potentially modifiable influences amenable to being addressed at the clinic encounter level: (a) cost and insurance coverage, (b) provider recommendation, (c) vaccination opportunity, (d) HPV and HPV vaccine knowledge, (e) vaccine safety concerns, and (f) HPV risk. See Table 2 for practice implications of these modifiable influences.

Research on HPV vaccine uptake began to take place soon after vaccine approval in 2006 and began to appear in publications in 2009. The studies reported a wide range of vaccine initiation, from $9 \%$ to $65 \%$, which may affect specific findings, but clearly demonstrates that high vaccination rates are possible. Research examining what is working in those areas with $65 \%$ uptake is needed, and also what is preventing vaccination in those areas with $9 \%$ vaccination rates. However, it should be noted that no study has demonstrated uptake levels in the United States that meet the $80 \%$ national tar- get immunization level set forth in Healthy People 2020 (www.healthypeople.gov).

Much of the research has focused on adolescents as patients and their parents as providers of consent, as vaccine initiation is recommended at age 11-12 years old. However, catch-up vaccination is recommended by the ACIP for all females through age 26 (Centers for Disease Control and Prevention, 2007), which encompasses a young adult group that will still benefit from the vaccine but may experience very different influences when facing vaccination. As adolescents transition into adulthood, they begin to make healthcare decisions without parental consent and often face fragmented healthcare delivery systems as they transit from pediatric providers to family, internal medicine, or reproductive health providers, and attend fewer preventive care visits (Rand et al., 2007). Further research is needed that focuses on the differences between the adolescent and young adult populations.

In addition, much of the clinical research has studied patients who are mostly insured, or are uninsured but under the age of 19 and therefore eligible for the Vaccines for Children program. Vaccines for Children is a federally funded, state-administered program that pays for many of the vaccines given to children in the United States. Young adults are not eligible for the Vaccines for Children program, leaving them with fewer cost coverage options for one of the most expensive vaccines on the market. The 19- to 26-year-old age group may face unstable insurance coverage as they move through school, unemployment, or the initial years of adult employment. Further research is needed that focuses on the uninsured population and ways to remove cost barriers.

The findings describing the association of provider characteristics with HPV vaccine initiation are limited in that they cannot explain if pediatric providers themselves, for example, are more likely to discuss, recommend, and 
carry vaccines, as is the case with other vaccines, or if relationships with pediatric providers have been more longstanding by adolescence, thereby increasing trust and influence, or even perhaps if those patients seeing pediatric providers are more amenable to vaccination.

Finally, because of the rapid changes occurring since the introduction of the HPV vaccine in 2006, the existing research may already be considered outdated. Initial research at the time of vaccine availability demonstrated high acceptability of the vaccine across a variety of populations (Brewer \& Fazekas, 2007), which has not translated into high vaccine uptake, preventing researchers from relying on acceptability research to develop predictors of HPV vaccine uptake. Since then, even intention to vaccinate has not been shown to be a reliable predictor of HPV vaccine uptake, with one study showing only $38 \%$ of parents who intended on vaccinating their daughter having done so a year later (Brewer et al., 2011). As a result, researchers will have to continue to study influences and predictors specific to uptake, without relying on acceptability or intention.

Since its approval, the vaccination process is rapidly progressing from its initial stages of availability to a time when the healthcare system has had the opportunity to become knowledgeable about the vaccine, establish systems and mechanisms for its delivery, and incorporate it into routine care. Nevertheless, we know that HPV vaccine uptake remains far below the Healthy People 2020 goal of $80 \%$ (www.healthypeople.gov), so much work remains for NPs.

\section{Acknowledgments}

Investigator support provided by the Health Promotion/Risk Reduction Interventions with Vulnerable Populations Training Grant (5 T32 NR007073-18). The authors would like to thank Maris Vinovskis, PhD for his input into previous versions of this manuscript.

\section{References}

Bosch, F. X., Lorincz, A., Muñoz, M., Meijer, C. J., \& Shah, K. V. (2002). The causal relation between human papillomavirus and cervical cancer. Journal of Clinical Pathology, 55(4), 244-265.

Brewer, N. T., \& Fazekas, K. I. (2007). Predictors of HPV vaccine acceptability: A theory-informed, systematic review. Preventive Medicine, 45, 107-1 14 doi:10.1016/j.ypmed.2007.05.013

Brewer, N. T., Gottlieb, S. L., Reiter, P. L., McRee, A., Liddon, N., Markowitz, L., \& Smith, J. S. (2011). Longitudinal predictors of human papillomavirus vaccine initiation among adolescent girls in a high-risk geographic area. Sexually Transmitted Diseases, 38(3), 197-204. doi:10.1097/OLQ.0b013e3181f12dbf

Caskey, R., Lindau, S. T., \& Alexander, G. C. (2009). Knowledge and early adoption of the HPV vaccine among girls and young women: Results of a national survey. Journal of Adolescent Health, 45(5), 453-462. doi:10.1016/j.jadohealth.2009.04.021
Centers for Disease Control and Prevention. (2007). Quadrivalent human papillomavirus vaccine: Recommendations of the advisory committee on immunization practices (ACIP). Morbidity and Mortality Weekly Report, 56(RR-2), 1-24.

Centers for Disease Control and Prevention. (2010). Adult vaccination coverage reported via NHIS. Retrieved from www.cdc.gov/vaccines/stats-surv/nhis/default.htm

Centers for Disease Control and Prevention. (2011). National and state vaccination coverage among adolescents aged 13 through 17 years-United States, 2010. Morbidity and Mortality Weekly Report, 60(33), 1117-1123.

Chao, C., Velicer, C., Slezak, J. M., \& Jacobsen, S. J. (2010). Correlates for human papillomavirus vaccination of adolescent girls and young women in a managed care organization. American Journal of Epidemiology, 171(3), 357-367. doi:10.1093/aje/kwp365

Conroy, K., Rosenthal, S. L., Zimet, G. D., Jin, Y., Bernstein, D. I., Glynn, S., \& Kahn, J. A. (2009). Human papillomavirus vaccine uptake, predictors of vaccination, and self-reported barriers to vaccination. Journal of Women's Health, 18(10), 1679-1686. doi:10.1089/jwh.2008.1329

Cook, R. L., Zhang, J., Mullins, J., Kauf, T., Brumback, B., Steingraber, H., \& Mallison, C. (2010). Factors associated with initiation and completion of human papillomavirus vaccine series among young women enrolled in Medicaid. Journal of Adolescent Health, 47(6), 596-599. doi:10.1016/j.jadohealth.2010.09.015

Dempsey, A. F., Abraham, L. M., Dalton, V., \& Ruffin, M. (2009). Understanding the reasons why mothers do or do not have their adolescen daughters vaccinated against human papillomavirus. Annals of Epidemiology, 19(8), 531-538. doi:10.1016/j.annepidem.2009.03.011

Dempsey, A., Cohn, L., Dalton, V., \& Ruffin, M. (2010). Patient and clinic factors associated with adolescent human papillomavirus vaccine utilization within a university-based health system. Vaccine, 28(4), 989-995. doi:10.1016/j.vaccine.2009.10.133

Dunne, E. F., Unger, E. R., Sternberg, M., McQuillan, G., Swan, D. C., Patel, S S., \& Markowitz, L. E. (2007). Prevalence of HPV infection among females in the United States. Journal of the American Medical Association, 297(8), 813-819. doi:10.1001/jama.297.8.813

Gerend, M. A., Weibley, E., \& Bland, H. (2009). Parental response to human papillomavirus vaccine availability: Uptake and intentions. Journal of Adolescent Health, 45(5), 528-531. doi:10.1016/j.jadohealth.2009.02.006

Gottlieb, S. L., Brewer, N. T., Sternberg, M. R., Smith, J. S., Ziarnowski, K., Liddon, N., \& Markowitz, L. E. (2009). Human papillomavirus vaccine initiation in an area with elevated rates of cervical cancer. Journal of Adolescent Health, 45(5), 430-437. doi:10.1016/j.jadohealth.2009.03.029

Guerry, S. L., De Rosa, C. J., Markowitz, L. E., Walker, S., Liddon, N., Kerndt, P. R., \& Gottlieb, S. L. (2011). Human papillomavirus vaccine initiation among adolescent girls in high-risk communities. Vaccine, 29, 2235-2241. doi:10.1016/j.vaccine.2011.01.052

Insinga, R. P., Dasbach, E. J., \& Elbasha, E. H. (2005). Assessing the annual economic burden of preventing and treating anogenital human papillomavirus-related disease in the US: Analytic framework and review of the literature. Pharmacoeconomics, 23(11), 1107-1122.

Jain, N., Euler, G. L., Shefer, A., Lu, P., Yankey, D., \& Markowitz, L. (2009). Human papillomavirus (HPV) awareness and vaccination initiation among women in the United States, national immunization survey-adult 2007. Preventive Medicine, 48(5), 426-431. doi:10.1016/j.ypmed.2008.11.010

Licht, A. S., Murphy, J. M., Hyland, A. J., Fix, B. V., Hawk, L. W., \& Mahoney, M. C. (2010). Is use of the HPV vaccine among female college students related to HPV knowledge and risk perception? Sexually Transmitted Infections, 86(1), 74-78. doi:10.1136/sti.2009.037705

Mathur, M., Mathur, V., \& Reichling, D. (2010). Participation in the decision to become vaccinated against human papillomavirus by California high school girls and the predictors of vaccine status. Journal of Pediatric Health Care, 24(1), 14-24. doi:10.1016/j.pedhc.2008.11.004

Moore, G. R., Crosby, R. A., Young, A., \& Charnigo, R. (2010). Low rates of free human papillomavirus vaccine uptake among young women. Sexual Health, 7(3), 287-290. doi:10.1071/SH09136 
Rand, C. M., Shone, L. P., Albertin, C., Auinger, P., Klein, J. D., \& Szilagyi, P. G. (2007). National health care visit patterns of adolescents. Archives of Pediatric and Adolescent Medicine, 161(3), 252-259. doi:10.1001/archpedi.161.3.252

Reiter, P. L., Cates, J. R., McRee, A. L., Gottlieb, S. L., Shafer, A., Smith, J. S., \& Brewer, M. T. (2010). Statewide HPV vaccine initiation among adolescent females in North Carolina. Sexually Transmitted Diseases, 37(12), 549-556. doi:10.1097/OLQ.0b013e3181d73bf8

Rosenthal, S. L., Weiss, T. W., Zimet, G. D., Ma, L., Good, M. B., \& Vichnin, M D. (2011). Predictors of HPV vaccine uptake among women aged 19-26: Importance of a physician's recommendation. Vaccine, 29(5), 890-895. doi:10.1016/j.vaccine.2009.12.063

Schluterman, N. H., Terplan, M., Lydecker, A. D., \& Tracy, J. K. (2011). Human papillomavirus (HPV) vaccine uptake and completion at an urban hospital. Vaccine, 29, 3767-3772. doi:10.1016/j.vaccine.2011.03.032

Slade, B. A., Leidel, L., Vellozzi, C., Woo, E. J., Hua, W., Sutherland, A., Izurieta, H. S., Ball, R., Miller, N., Braun, M. M., Markowitz, L. E., \&
Iskander, J. (2009). Postlicensure safety surveillance for quadrivalent human papillomavirus recombinant vaccine. JAMA, 302 (7), 750-759.

Small, S. L., \& Patel, D. A. (2012). Impact of HPV vaccine availability on uptake. Journal for Nurse Practitioners, 8(1), 61-66.

Zimet, G. D., Weiss, T. W., Rosenthal, S. L., Good, M. B., \& Vichnin, M. D. (2010). Reasons for non-vaccination against HPV and future vaccination intentions among 19-26 year-old women. BMC Women's Health, 10(27), $1-6$.

\section{Supporting Information}

Additional Supporting Information may be found in the online version of this article at the publisher's web site:

Table S1. Summary of studies: modifiable influences on HPV vaccine uptake. 